\title{
Hydroxychloroquine enhances efferocytosis and modulates the gut microbiome in mice with pristane-induced lupus
}

\section{Ting-Yin Xue}

China Medical University

Fei-Hung Hsieh

China Medical University

Jun-Chieh J. Tsay

New York University School of Medicine

Hsin-Yi Peng

China Medical University

Yen-Chi Tsao

China Medical University

Mei-Chin Yin

Asia University

Che-Yi Chao

Asia University

Shu-Yao Tsai

Asia University

Chung-Ping Lin

Asia University

Jiunn-Horng Chen

China Medical University Hospital

Chung-Ming Huang

China Medical University Hospital

Hen-Hong Chang

China Medical University Hospital

Zsuzsa Szondy

University of Debrecen

Gregory J Tsay ( $\nabla_{\text {jjtsay@mail.cmu.edu.tw ) }}$

China Medical University 
Keywords: hydroxychloroquine, efferocytosis, pristine-induced lupus, autoimmune diseases, microbiome Posted Date: April 8th, 2022

DOI: https://doi.org/10.21203/rs.3.rs-1094062/v2

License: (c) (1) This work is licensed under a Creative Commons Attribution 4.0 International License. Read Full License 


\section{Abstract}

Background An important contributor to the pathogenesis of the autoimmune disease systemic lupus erythematosus (SLE) is the impaired clearance of apoptotic cells (efferocytosis) and increasing evidence implicates microbiota dysbiosis as another important player. Hydroxychloroquine (HCQ) is frequently prescribed for the treatment of SLE. Here, we evaluate changes in efferocytosis and the gut microbiome in mice with pristane-induced lupus (PIL) by HCQ administration.

Methods PIL mice were studied with or without HCQ and/or resveratrol (RESV). Efferocytosis was determined in RAW 264.7 cells and peritoneal macrophages from mouse ascites fluid. The gut microbiome was analyzed using Illumina sequencing.

Results Both HCQ and RESV enhanced efferocytosis. HCQ also significantly suppressed ascites production of proinflammatory cytokines interleukin (IL)- 6 and tumor necrosis factor alpha (TNF-a). The Firmicutes/Bacteroidetes $(F / B)$ ratio was significantly decreased in PIL mice compared with untreated controls $(p<0.05)$. The $F / B$ ratio in PIL mice was increased by HCQ alone and significantly increased by HCQ combined with RESV.

Conclusions In this study, HCQ and RESV enhanced efferocytosis in both RAW 264.7 cells and peritoneal macrophages of PIL mice. The pristane-induced reduction in the F/B ratio was restored by HCQ treatment. SLE treatment should consider the role of the gut microbiome.

\section{Introduction}

The etiology of systemic lupus erythematosus (SLE) is unclear, although it is well known that B-cell abnormalities, autoantibodies against nuclear antigens and immune complex deposition are crucial for its pathogenesis. ${ }^{1}$ Treatment for SLE is generally based on nonspecific immunosuppression. There is no cure for SLE and complete long-term remissions are rare. ${ }^{2}$ Notably, the efficient clearance of apoptotic cells (efferocytosis) is critical for maintaining tissue homeostasis and regulating innate immunity; ${ }^{3}$ impaired elimination of apoptotic cells and debris has been found to increase the susceptibility for developing SLE in both mice and humans. ${ }^{4,5}$

The gut microbiome is responsible for metabolic, immunological, structural and neurological functions. ${ }^{6}$ In particular, microbiota dysbiosis is implicated in the initiation and progression of autoimmune diseases ${ }^{7}$ and in the pathogenesis of systemic autoimmune diseases. ${ }^{8-10}$ Alterations in the gut microbiome may even correlate with SLE disease activities, although research has yet to define the exact roles of symbiotic and pathogenic microbes. ${ }^{10}$ Elucidating the roles of the gut microbiome in SLE is expected to provide opportunities for potential therapies. ${ }^{11}$ Interestingly, patients with SLE exhibit intestinal dysbiosis, characterized by a reduced Firmicutes/Bacteroidetes $(F / B)$ ratio, ${ }^{9,12}$ which suggests that targeting the $F / B$ ratio would be beneficial in SLE treatment. 
The immunomodulator hydroxychloroquine (HCQ) is the mainstay of treatment for autoimmune diseases such as SLE and Sjögren's syndrome. ${ }^{13}$ Advantages associated with HCQ include its good tolerability, its inexpensiveness and lower toxicity compared with other antirheumatic drugs. ${ }^{14}$ Several variables appear to be responsible for the beneficial effects of HCQ: its alteration of the lysosomal $\mathrm{pH}$ with a consequent reduction in antigen presentation; ${ }^{15,16}$ its impairment of the Toll-like receptor (TLR) function; ${ }^{17}$ its direct interaction with nucleic acids and its ability to inhibit interferon beta (IFN- $\beta$ ) production by blocking the cyclic GMP-AMP synthase (cGAS) - stimulator of interferon genes (STING) pathway. ${ }^{18-20}$ Notably, the effects of HCQ upon efferocytosis have not been explored until now.

Pristane-induced lupus (PIL) is widely used as a model of human SLE and is characterized by typical autoantibodies, clinical manifestations, organ involvement and arthritis similar to what is observed in SLE. ${ }^{21,22}$ Pristane-induced murine lupus is generally regarded as appropriate for examining the influence of various therapeutic interventions in vivo. We therefore used this model to study the therapeutic effects of HCQ.

Resveratrol (RESV) is a natural polyphenolic compound that is present in grapes, some berries and red wine. The antioxidant and anti-inflammatory properties of RESV have been shown to improve human health and to protect against cancer, diabetes, and Alzheimer's disease, amongst other disorders. ${ }^{23,24}$ The anti-inflammatory effect of RESV justified its addition to this study as a control.

This study aimed to investigate efferocytosis and changes in the gut microbiome in PIL mice treated with and without HCQ and RESV. We found that HCQ has beneficial effects on efferocytosis and the gut microbiome.

\section{Results}

\section{Efferocytosis in RAW 264.7 cells and peritoneal macrophages of PIL mice}

To determine appropriate doses of HCQ and RESV for the study, RAW 264.7 cells were incubated with HCQ and RESV at concentrations ranging from 1 to $30 \mu \mathrm{M}$ and cell viability was assessed by MTT testing $24 \mathrm{~h}$ later (Supplementary Fig. 1). HCQ at concentrations of 10, 15 and $20 \mu \mathrm{M}$ and RESV at concentrations of 1,5 and $10 \mu \mathrm{M}$ were chosen for evaluation of efferocytosis by MTT testing. Both HCQ and RESV significantly enhanced efferocytosis in RAW 264.7 cells in a dose-dependent manner (Fig. 2A and 2B). Pristane significantly decreased efferocytosis in the PIL-alone group (Fig. 2C). Both HCQ and/or RESV enhanced efferocytosis compared with PIL alone (Fig. 2C).

\section{Analysis of autoantibodies and cytokines in PIL mice}

Serum samples were collected from all mice for ANA and anti-dsDNA testing at 1, 3 and 6 months; ascites fluid was collected from all mice at 6 months for analysis of TNF-a and IL- 6 levels. In the PIL 
mice, pristane-induced increases in ANA titers were observed at 1 month and titers continued to increase until 6 months. At 6 months after pristane induction, ANA titers had increased from $139.7 \mathrm{U} / \mathrm{ml}$ at baseline in untreated control mice to $429.5 \mathrm{U} / \mathrm{ml}$ in PIL mice (Fig. 3A). Pristane-induced increases in antidsDNA antibodies were first observed at 3 months and continued to increase until 6 months (Fig. 3B). Treatments with HCQ and/or RESV did not change ANA and DNA titers.

At 6 months after PIL induction, TNF-a levels in ascites fluid were increased by pristane and decreased by HCQ or RESV treatment (Fig. 3C). Pristane also increased IL- 6 levels in ascites fluid at 6 months after PIL induction, whereas HCQ and/or RESV significantly decreased IL-6 levels (Fig. 3D).

\section{Analysis of the gut microbiome}

In this study, we investigated changes in the microbiome between the PIL mice and those treated with HCQ alone or in combination with RESV. We noted between-group differences at the specific phyla level. Pristane decreased relative abundances of Firmicutes and increased abundances of Bacteroidetes in PIL mice (group D, Fig. 4A-C) compared with untreated controls (group E, Fig. 4A-C). HCQ showed a trend towards an increase in Firmicutes and a decrease in Bacteroidetes (group C, Fig. 4A-C) compared with pristane alone (group D, Fig. 4A-C). Similar changes were seen in RESV-treated PIL mice (group B, Fig. 4AC) compared with PIL alone (group D, Fig. 4A-C). Furthermore, treatments with both HCQ and RESV (group A, Fig. 4A-C) significantly increased abundances of Firmicutes and decreased abundances of Bacteroides compared with PIL alone (group D, Fig. 4A-C). We observed a significant decrease in the $F / B$ ratio for PIL mice compared with untreated controls (Fig. 4D), while treatment with HCQ in combination with RESV in PIL mice significantly increased the $F / B$ ratio compared with PIL alone (Fig. 4D).

Alpha-diversity measures in fecal samples based on the Shannon index revealed between-group differences in mice treated with RESV (Groups A and B, Fig. 5A) compared with mice that were not (Groups C, D and E, Fig. 5A; $p=0.023$, Fig. 5A). There was no statistically significant difference in the Shannon Index between each group (Groups A-E, Fig. 5B). PCOA assessments of fecal samples demonstrated a markedly different microbiome distribution in mice that received RESV (Groups A and B, Fig. 5 C) versus those that did not (Groups C, D and E, Fig. 5C).

LEfSe of bacterial biomarkers showed that treatment with HCQ increased the abundance of Bacillales, Aerococcus, Staphylococcaceae, Staphylococcus, and Jeotgalicoccus (Supplementary Fig. 2). Treatment with RESV increased the abundance of Haemophilus, Pasteurellaceae, and Pasteurellales, while treatment with HCQ in combination with RESV increased the abundance of Lactobacillus, Lactobacillaceae, and Lactobacillales.

\section{Discussion}

We have demonstrated for the first time that HCQ can modify the gut microbiome, increase efferocytosis in macrophages from both the RAW 264.7 mouse cell line and peritoneal macrophages of PIL mice, and suppress the production of TNF- $a$ and IL-6 from ascites fluid of PIL mice. Our results provide insights into 
the novel anti-inflammatory mechanisms of HCQ. It is established that acute gut infections can permanently affect tissue immunity ${ }^{7}$ and that the gut microbiome plays a role in drug metabolism. ${ }^{11}$ The multiple effects induced by HCQ regarding modification of the microbiome, increase in efferocytosis and suppression of proinflammatory cytokines provide further beneficial information for its long-term therapy for SLE. Currently, the main therapies for SLE are immunosuppressive drugs and glucocorticoids, all of which cause many adverse side effects and are not recommended for use as long-term treatments.

HCQ has been widely used to treat many autoimmune rheumatic diseases including SLE, rheumatoid arthritis and Sjögren's syndrome, for its anti-inflammatory and immunomodulatory effects and is considered to be one of the safest antirheumatic agents with very infrequent serious side effects. ${ }^{14}$ Beneficial effects of $\mathrm{HCQ}$ include the alteration of lysosomal $\mathrm{pH}^{25} \mathrm{TLR}^{2}$ function, ${ }^{13}$ and inhibition of type I IFN. ${ }^{19,20}$ We have described a novel effect of HCQ, whereby the drug enhances efferocytosis and thus attenuates SLE-associated inflammation. There is a huge unmet need for effective SLE therapy. Drugs such as HCQ that enhance efferocytosis may have therapeutic potential for SLE.

Previous research has described how chloroquine inhibits the production of TNF-a, IL-1 $\beta$ and IL-6 in human U937 and THP-1 macrophages. ${ }^{26}$ Moreover, we have found that HCQ inhibits the production of TNF- $a$ and IL- 6 in PIL mice. Patients with SLE have elevated serum levels of IL- 6 and TNF-a, which correlate with disease activity. ${ }^{27-29}$ In addition to enhancing efferocytosis, HCQ downregulates IL- 6 and TNF-a levels and thus helps to resolve SLE inflammation.

Murine studies have explored the relationship between the microbiome and SLE. ${ }^{30}$ The PIL mouse model offers the means to study lupus in humans. ${ }^{31}$ One study using female MRL/lpr mice found that they had a distinct bacterial microbiota and species diversity compared with healthy controls; the lupus-prone mice exhibited depleted Lactobacillus and more abundant Lachnospraceae. ${ }^{32}$ Similarly, we found that treatment with HCQ and RESV resulted in unique microbiome expression with lactobacillus. Lactobacillus species constitute a significant component of human and animal microbiota in the digestive system and exhibit a mutualistic relationship with the body to protect the host against potential invasions by pathogens. Study evidence has detailed how the gut microbiome is perturbed in rheumatoid arthritis, with the depletion of Haemophilus spp in particular. ${ }^{33}$ The findings from our study showing that RESV treatment in PIL mice is associated with a unique microbiome with Haemophilus (Supplementary Fig. 2) suggests that RESV therapy is beneficial in PIL mice.

In our study, pristane-induced decreases in the gut $F / B$ ratio were restored after HCQ treatment, which improved microbiome alterations in PIL mice. The $F / B$ ratio from the gut microbiome has previously been shown to be associated with systemic inflammation. ${ }^{34,35}$ Growing evidence suggests that intestinal dysbiosis may be an important part of the pathogenesis of autoimmune diseases such as rheumatoid arthritis, inflammatory bowel disease and SLE. Future treatment for SLE should consider the role of the gut microbiome. 
Some limitations exist in our study. Firstly, we did not use human macrophages during HCQ treatment to study the processes involving efferocytosis, the cytokine profiles and the gut microbiome. Future experiments in humans will be necessary to determine these effects with HCQ treatment. Secondly, we did not determine whether HCQ affects survival of HCQ-treated mice. Moreover, longer durations of treatment are necessary for future studies.

In conclusion, HCQ enhances efferocytosis, suppresses the production of IL-6 and TNF-a and modulates the gut microbiome in PIL mice. Our study provides novel insights into the potential role of HCQ for the treatment of SLE.

\section{Methods}

\section{Cell culture}

RAW 264.7 cells were purchased from the Bioresource Collection and Research Center (Hsinchu, Taiwan) and were cultured in Dulbecco's Modified Eagle Medium (DMEM; Gibco, Grand Island, NY) containing 10\% fetal bovine serum (FBS; HyClone, Logan, UT, USA). EL4 cells were purchased from Bioresource Collection and Research Center and were cultured in DMEM containing 10\% horse serum (HyClone). Human keratinocyte cells (HaCaT) were gifted by Professor Jen-Hung Yang from the Department of Dermatology in Chung Shan Medical University (Taichung, Taiwan) and were cultured in DMEM containing 10\% FBS.

\section{Cell viability}

RAW 264.7 cells were treated with 1, 5, 10, 15, 20, or $30 \mu \mathrm{M}$ of RESV (Sigma Chemical, St. Louis, MO, USA) or HCQ (Cayman Chemical, Ann Arbor, Michigan) for $24 \mathrm{~h}$ each and were analyzed for cell viability using the 3-(4,5-dimethylthiazol-2-yl)-2,5-diphenyltetrazolium bromide (MTT; BioVision, Milpitas, CA, USA) assay.

\section{Efferocytosis assay}

RAW 264.7 cells were stained with carboxyfluorescein succinimidyl ester (CFSE; eBioscience, Santa Clara, CA, USA) and treated with HCQ or RESV for $24 \mathrm{~h}$. EL4 cells were labeled with MitoTracker Deep Red FM (Thermo Fisher Scientific Inc., Waltham, MA, USA) and exposed to UVB radiation $400 \mathrm{~J} / \mathrm{m}^{2}$. After $24 \mathrm{~h}$ of UVB-induced apoptosis, EL4 cells were co-cultured with RAW cells at a ratio of $10: 1$ at $37^{\circ} \mathrm{C}$ for $1 \mathrm{~h} .{ }^{36}$ After both cell lines were washed with PBS, the cells were scratched and fluorescent intensity was analyzed using a BD FACSCelesta flow cytometer (BD Biosciences, Franklin Lakes, NJ, USA). Peritoneal macrophages were harvested from PIL mice and examined with the same efferocytosis assay. ${ }^{37}$

\section{Experimental SLE design}

Fifty-eight 6-week-old female BALB/c mice were purchased from LASCO (Taipei, Taiwan) and divided into five groups: Group A, PIL + RESV + HCQ ( $n=12)$; Group B, PIL + RESV $(n=12)$; Group C, PIL + HCQ $(n=12)$; Group D, PIL monotherapy $(n=10)$; and Group E, untreated controls $(n=12)$ (Fig. 1). The project was 
approved by the Institutional Animal Care and Use Committee of China Medical University Hospital, Taichung, Taiwan (CMUIACUC-2018-282-1, June 2018-June 2019). The study is reported in accordance with ARRIVE guidelines. Mice were housed in the Laboratory Animal Service Center in CMU. PIL was induced by a single intraperitoneal injection of pristane $0.5 \mathrm{ml}$ (Sigma Chemical, St. Louis, MO, USA). HCQ (ACROS, Geel, Belgium) $3 \mathrm{mg} / \mathrm{kg} /$ day that started from day 1 of PIL induction and lasted for 180 consecutive days; RESV (Tokyo Chemical Industry Co., Ltd., Tokyo, Japan) 50 mg/kg/day commenced from 30 days before PIL induction and lasted for 210 consecutive days. At 180 days after PIL induction, all mice were euthanized with Zoletil 50 and ascites fluid and serum were collected for anti-dsDNA, antinuclear antibodies (ANA), tumor necrosis factor alpha (TNF- $a$ ) and interleukin (IL)- 6 analysis by ELISA and efferocytosis. ${ }^{36}$

\section{Enzyme-linked immunosorbent assay (ELISA) analysis}

Mice were injected with $5 \mathrm{ml}$ of PBS into the peritoneal cavity. Ascites fluid was analyzed by ELISA, ${ }^{36}$ as per the manufacturer's procedure. Mouse TNF- $\alpha$ and IL-6 ELISA kits were purchased from BioLegend (San Diego, CA, USA). An ANA ELISA kit was purchased from Alpha Diagnostic Intl. (San Antonio, TX, USA). The mouse anti-dsDNA assay kit was purchased from Chondrex Inc. (Redmond, WA, USA).

\section{Fecal 16S rDNA sequencing and OTU analysis and annotation}

Fecal samples were collected from mice and stored at $-80^{\circ} \mathrm{C}$. Fecal DNA was extracted using the QIAamp PowerFecal Pro DNA Kit (Qiagen, Venlo, The Netherlands), following the standard protocol. OTU analysis and annotation was performed by Welgene Biotech Co., Ltd. (Taipei, Taiwan) (Supplementary material).

\section{Statistical analysis}

Statistically significant differences between groups were determined using the two-tailed unpaired $t$-test. Reported data were verified by at least three separate experiments and are represented as the mean \pm SEM or mean \pm SD. A p-value of $<0.05$ was considered to be significant. Analyses of graphs and statistics were performed using GraphPad Prism software version 6.

We confirm that all experiments were performed in accordance with relevant guidelines and regulations.

\section{Abbreviations}

SLE: Systemic lupus erythematosus; HCQ: Hydroxychloroquine; PIL: Pristane-induced lupus; RESV: Resveratrol; IL: Interleukin; TNF-a: Tumor necrosis factor alpha; F/B ratio: Firmicutes/Bacteroidetes (F/B) ratio; TLR: Toll-like receptor; IFN- $\beta$ : Inhibit interferon beta; cGAS: Cyclic GMP-AMP synthase; STING: Stimulator of interferon genes

\section{Declarations}




\section{Acknowledgments}

The authors would like to thank Edward K.L. Chan, PhD for his critical reading and revision of our manuscript. Dr. Chan is a professor in the Department of Oral Biology at the UF College of Dentistry and a professor in the Department of Anatomy and Cell Biology at the UF College of Medicine. We also thank Iona J. $\mathrm{M}^{\mathrm{ac}}$ Donald from China Medical University for her editing input.

\section{Authors' contributions}

All authors made substantive intellectual contributions to this study to qualify as authors. GJT designed and supervised the experiments and wrote the paper. TYX and FHH performed the experiments and participated in drafting the paper. Data were analyzed and written in part by JCJT. HYP and YCT performed the experiments.

$\mathrm{JHC}$ and $\mathrm{CMH}$ collected and interpreted the data. MCY, CYC, SYT, CPL, HHC and ZS conceived the study and also analyzed and interpreted the data. All authors have read and approved the final manuscript.

\section{Data Availability}

The datasets generated during and/or analyzed during the current study are available by request. The data that support the findings of this study are available on request from the corresponding author, GJT.

\section{Additional Information}

\section{Competing Interests}

All the authors declare that the research was conducted in the absence of any commercial or financial relationships that could be construed as a potential conflict of interest.

\section{Funding}

This study was supported by grants from the Ministry of Science and Technology, Taiwan (MOST-1042314-B-039-045, MOST-105-2911-I-039-504, MOST-105-2314-B-039-047, MOST-106-2911-I-039-501, MOST-107-2314-B-039-050, MOST-108-2314-B-039-019), and from China Medical University Hospital (DMR-106-188, DMR-108-183, DMR-109-206, DMR-111-199).

\section{Ethics approval and consent to participate}

The project was approved by the Institutional Animal Care and Use Committee of China Medical University Hospital, Taichung, Taiwan (CMUIACUC-2018-282-1, June 2018-June 2019).

\section{Consent for publication}

Not applicable 


\section{Author details}

${ }^{1}$ Research and Development Center for Immunology, China Medical University, Taichung, Taiwan

${ }^{2}$ Division of Pulmonary and Critical Care Medicine, New York University School of Medicine, New York, USA

${ }^{3}$ Division of Pulmonary and Critical Care Medicine, VA New York Harbor Healthcare System, New York, USA

${ }^{4}$ Autoimmune Disease Laboratory, China Medical University Hospital, Taichung, Taiwan

${ }^{5}$ Department of Food Nutrition and Health Biotechnology, Asia University, Taichung, Taiwan

${ }^{6}$ Department of Medical Research, China Medical University Hospital, Taichung, Taiwan

${ }^{7}$ Division of Immunology and Rheumatology, Department of Internal Medicine, China Medical University Hospital, Taichung, Taiwan

${ }^{8}$ College of Medicine, China Medical University, Taichung, Taiwan

${ }^{9}$ Department of Chinese Medicine, China Medical University Hospital, Taichung, Taiwan

${ }^{10}$ Graduate Institute of Integrated Medicine, Graduate Institute of Acupuncture Science, College of Chinese Medicine, China Medical University, Taichung, Taiwan

${ }^{11}$ Section of Dental Biochemistry, Department of Biochemistry and Molecular Biology, Faculty of Dentistry, University of Debrecen, Debrecen, Hungary.

\section{References}

1. Tsokos, G. C. Systemic lupus erythematosus. The New England journal of medicine 365, 2110-2121, doi:10.1056/NEJMra1100359 (2011).

2. Wei, S. et al. Pin1-Targeted Therapy for Systemic Lupus Erythematosus. Arthritis \& rheumatology (Hoboken, N.J.) 68, 2503-2513, doi:10.1002/art.39741 (2016).

3. Szondy, Z., Garabuczi, E., Joós, G., Tsay, G. J. \& Sarang, Z. Impaired clearance of apoptotic cells in chronic inflammatory diseases: therapeutic implications. Frontiers in immunology 5, 354, doi:10.3389/fimmu.2014.00354 (2014).

4. Herrada, A. A. et al. Innate immune cells' contribution to systemic lupus erythematosus. Frontiers in immunology 10, 772 (2019).

5. Huang, W. N., Tso, T. K., Wu, H. C., Yang, H. F. \& Tsay, G. J. Impaired phagocytosis of apoptotic cell material in serologically active clinically quiescent patients with systemic lupus erythematosis. 
International journal of rheumatic diseases 19, 1310-1316, doi:10.1111/1756-185x.12826 (2016).

6. Adak, A. \& Khan, M. R. An insight into gut microbiota and its functionalities. Cellular and Molecular Life Sciences 76, 473-493 (2019).

7. Belkaid, Y. \& Hand, T. W. Role of the microbiota in immunity and inflammation. Cell 157, 121-141 (2014).

8. Hevia, A. et al. Intestinal dysbiosis associated with systemic lupus erythematosus. mBio 5, e0154801514, doi:10.1128/mBio.01548-14 (2014).

9. Manfredo Vieira, S. et al. Translocation of a gut pathobiont drives autoimmunity in mice and humans. Science (New York, N.Y.) 359, 1156-1161, doi:10.1126/science.aar7201 (2018).

10. Katz-Agranov, N. \& Zandman-Goddard, G. The microbiome and systemic lupus erythematosus. Immunologic research 65, 432-437 (2017).

11. Holmes, E. et al. Therapeutic modulation of microbiota-host metabolic interactions. Science translational medicine 4, 137rv136-137rv136 (2012).

12. López, P. et al. Th17 responses and natural IgM antibodies are related to gut microbiota composition in systemic lupus erythematosus patients. Scientific reports 6, 1-12 (2016).

13. An, J., Minie, M., Sasaki, T., Woodward, J. J. \& Elkon, K. B. Antimalarial drugs as immune modulators: new mechanisms for old drugs. Annual review of medicine 68, 317-330 (2017).

14. Ponticelli, C. \& Moroni, G. Hydroxychloroquine in systemic lupus erythematosus (SLE). Expert opinion on drug safety 16, 411-419 (2017).

15. Ohkuma, S. \& Poole, B. Fluorescence probe measurement of the intralysosomal pH in living cells and the perturbation of $\mathrm{pH}$ by various agents. Proceedings of the National Academy of Sciences 75, 3327-3331 (1978).

16. Homewood, C., Warhurst, D., Peters, W. \& Baggaley, V. Lysosomes, $\mathrm{p} \mathrm{H}$ and the Anti-malarial Action of Chloroquine. Nature 235, 50-52 (1972).

17. Boulé, M. W. et al. Toll-like receptor 9 -dependent and-independent dendritic cell activation by chromatin-immunoglobulin G complexes. The Journal of experimental medicine 199, 1631-1640 (2004).

18. Sacre, K., Criswell, L. A. \& McCune, J. M. Hydroxychloroquine is associated with impaired interferonalpha and tumor necrosis factor-alpha production by plasmacytoid dendritic cells in systemic lupus erythematosus. Arthritis research \& therapy 14, 1-10 (2012).

19. An, J., Woodward, J. J., Sasaki, T., Minie, M. \& Elkon, K. B. Cutting edge: Antimalarial drugs inhibit IFN- $\beta$ production through blockade of cyclic GMP-AMP synthase-DNA interaction. Journal of immunology (Baltimore, Md.: 1950) 194, 4089-4093, doi:10.4049/jimmunol.1402793 (2015).

20. An, J. et al. Inhibition of cyclic GMP-AMP synthase using a novel antimalarial drug derivative in Trex1-deficient mice. Arthritis \& Rheumatology 70, 1807-1819 (2018).

21. Calvani, N. et al. Induction of apoptosis by the hydrocarbon oil pristane: implications for pristaneinduced lupus. The Journal of Immunology 175, 4777-4782 (2005). 
22. Leiss, H. et al. Pristane-induced lupus as a model of human lupus arthritis: evolvement of autoantibodies, internal organ and joint inflammation. Lupus 22, 778-792 (2013).

23. Baur, J. A. et al. Resveratrol improves health and survival of mice on a high-calorie diet. Nature 444, 337-342 (2006).

24. Berman, A. Y., Motechin, R. A., Wiesenfeld, M. Y. \& Holz, M. K. The therapeutic potential of resveratrol: a review of clinical trials. NPJ precision oncology 1, 1-9 (2017).

25. Fox, R. I. in Seminars in arthritis and rheumatism. 82-91 (Elsevier).

26. Jang, C., Choi, J., Byun, M. \& Jue, D. Chloroquine inhibits production of TNF-a, IL-1b and IL-6 from lipopolysaccharide-stimulated human monocytes/macrophages by different modes. Rheumatology 45, 703-710 (2006).

27. Ripley, B., Goncalves, B., Isenberg, D., Latchman, D. \& Rahman, A. Raised levels of interleukin 6 in systemic lupus erythematosus correlate with anaemia. Annals of the Rheumatic Diseases 64, 849853 (2005).

28. Aringer, M. \& Smolen, J. S. The role of tumor necrosis factor-alpha in systemic lupus erythematosus. Arthritis research \& therapy 10,1-8 (2008).

29. Linker-Israeli, M. et al. Elevated levels of endogenous IL-6 in systemic lupus erythematosus. A putative role in pathogenesis. The Journal of Immunology 147, 117-123 (1991).

30. Hanayama, R. et al. Autoimmune disease and impaired uptake of apoptotic cells in MFG-E8-deficient mice. Science (New York, N.Y.) 304, 1147-1150, doi:10.1126/science.1094359 (2004).

31. Patten, C. et al. Characterization of pristane-induced arthritis, a murine model of chronic disease: Response to antirheumatic agents, expression of joint cytokines, and immunopathology. Arthritis \& Rheumatism: Official Journal of the American College of Rheumatology 50, 3334-3345 (2004).

32. Zhang, H., Liao, X., Sparks, J. B. \& Luo, X. M. Dynamics of gut microbiota in autoimmune lupus. Applied and environmental microbiology 80, 7551-7560 (2014).

33. Zhang, X. et al. The oral and gut microbiomes are perturbed in rheumatoid arthritis and partly normalized after treatment. Nature medicine 21, 895-905 (2015).

34. Verdam, F. J. et al. Human intestinal microbiota composition is associated with local and systemic inflammation in obesity. Obesity 21, E607-E615 (2013).

35. Stojanov, S., Berlec, A. \& Štrukelj, B. The influence of probiotics on the Firmicutes/Bacteroidetes ratio in the treatment of obesity and inflammatory bowel disease. Microorganisms 8, 1715 (2020).

36. Yen, J.-H. et al. Daidzein enhances efferocytosis via transglutaminase 2 and augmentation of Rac1 activity. Molecular immunology 60, 135-142 (2014).

37. Li, S. et al. Defective phagocytosis of apoptotic cells by macrophages in atherosclerotic lesions of ob/ob mice and reversal by a fish oil diet. Circulation research 105, 1072-1082 (2009).

\section{Supplemental Data}

Supplementary Figures 1 and 2 are not available with this version 


\section{Figures}

\begin{tabular}{|c|c|c|c|c|}
\multicolumn{4}{c|}{ Pristane-induced lupus (PIL) } \\
\cline { 1 - 1 } & PIL + RESV & PIL + HCQ & PIL & Normal (Untreated) \\
\cline { 3 - 5 } & Group B & Group C & Group D & Group E
\end{tabular}

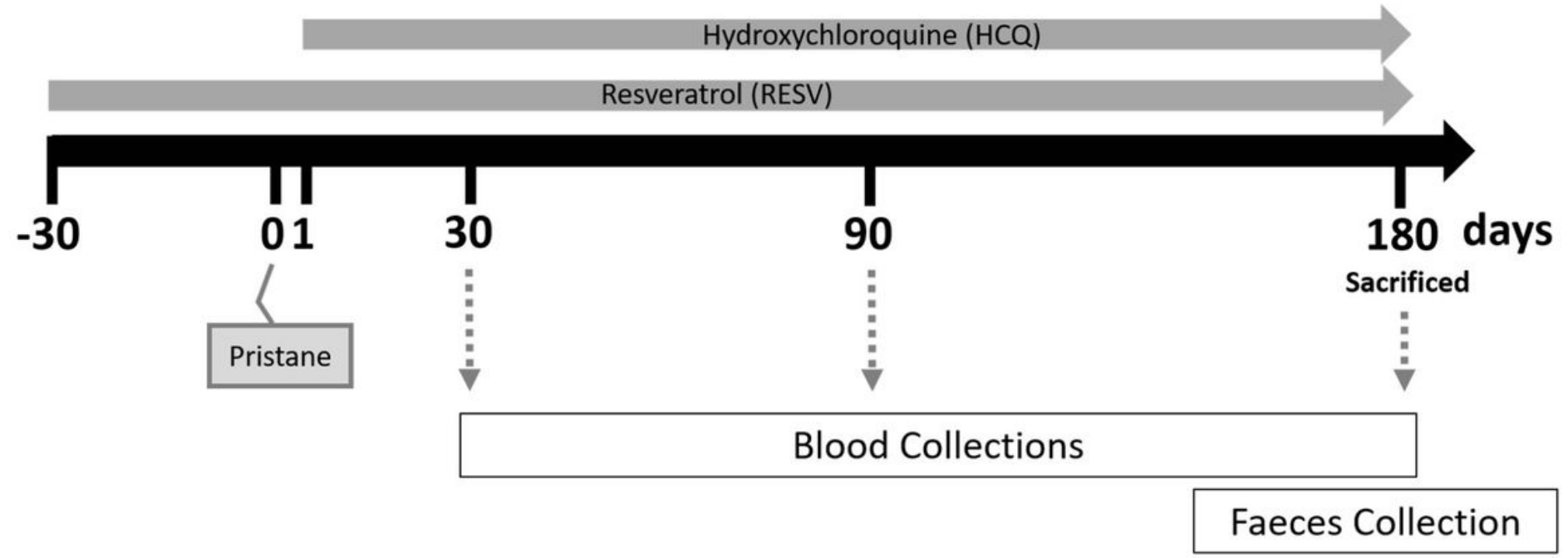

\section{Figure 1}

Experimental design for animal studies.

Fifty-eight 6-week-old female BALB/c mice were divided into five groups. Group A $(n=12)$ treated with pristane (PIL) was given resveratrol (RESV) starting at -30 days and hydroxychloroquine (HCQ) starting on day 1 (PIL+RESV+HCQ). Group B $(n=12)$ PIL-treated mice were given only RESV (PIL+RESV). Group C $(n=12)$ PIL-treated mice were given only HCQ (PIL+HCQ). Group D $(n=10)$ consisted of PIL-treated mice only (PIL). Group $E(n=12)$ received no treatment (untreated control mice). HCQ $3 \mathrm{mg} / \mathrm{kg} /$ day started from day 1 of PIL induction and lasted for 180 consecutive days; RESV $50 \mathrm{mg} / \mathrm{kg} /$ day commenced from 30 days before PIL induction and lasted for 210 consecutive days. Serum and fecal samples were collected from all study groups on certain days, as indicated. 

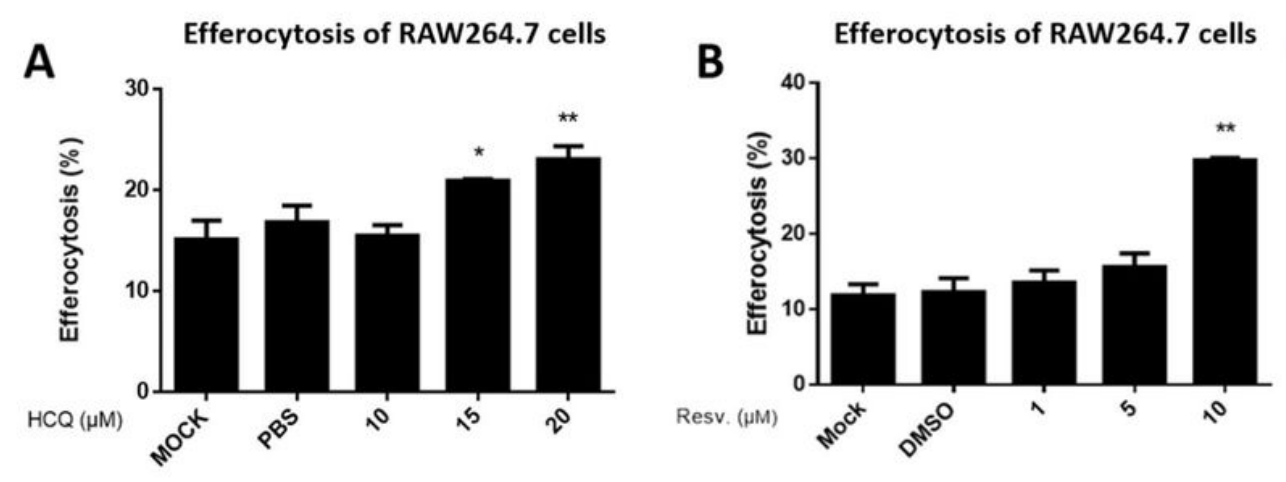

Efferocytosis of peritoneal macrophage

C

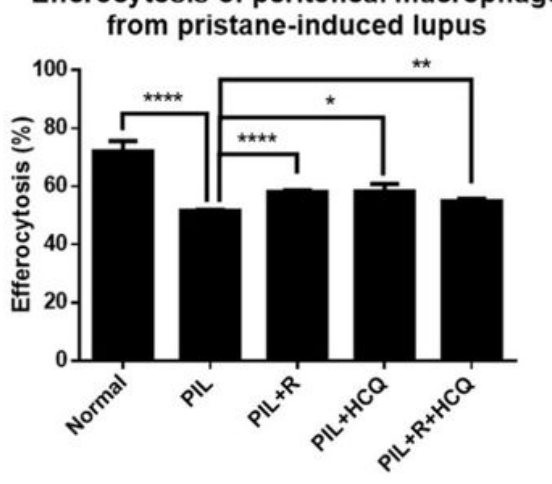

Figure 2

Analysis of efferocytosis in RAW cells and peritoneal macrophages from ascites of mice with PIL.

After treatment with HCQ or RESV, RAW 264.7 cells were co-cultured with apoptotic cells to study efferocytosis by flow cytometry. Both HCQ and RESV significantly enhanced efferocytosis in RAW 264.7 cells in a dose-dependent manner (Fig. 2A and 2B). Peritoneal macrophages were collected at 6 months after pristane induction. Pristane significantly decreased efferocytosis (Fig. 2C), while both HCQ and/or RESV enhanced efferocytosis (Fig. 2C). 
A
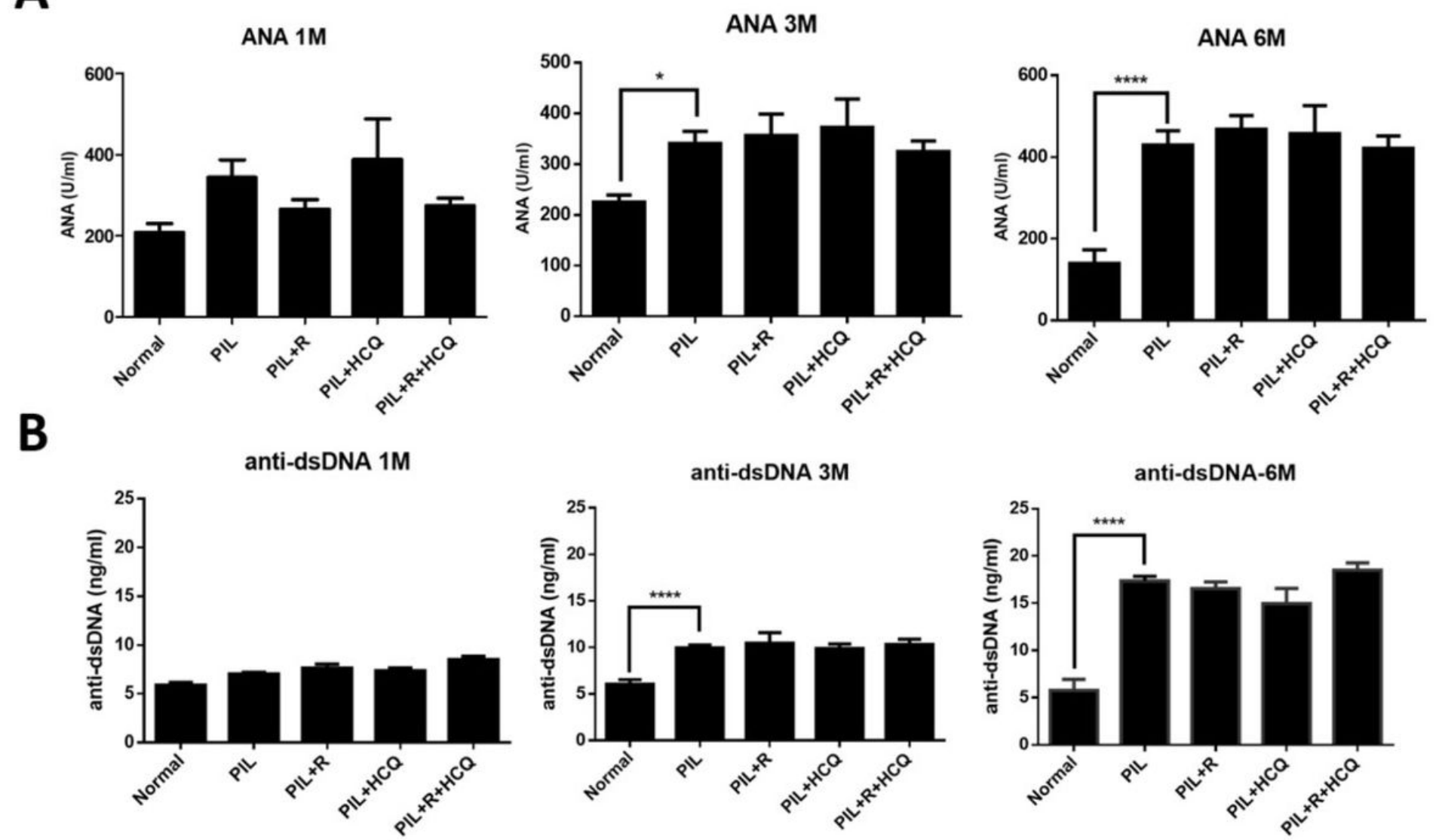

C

TNF- $\alpha$ level in Ascetic Filuid

D

IL-6 in Ascetic fluid
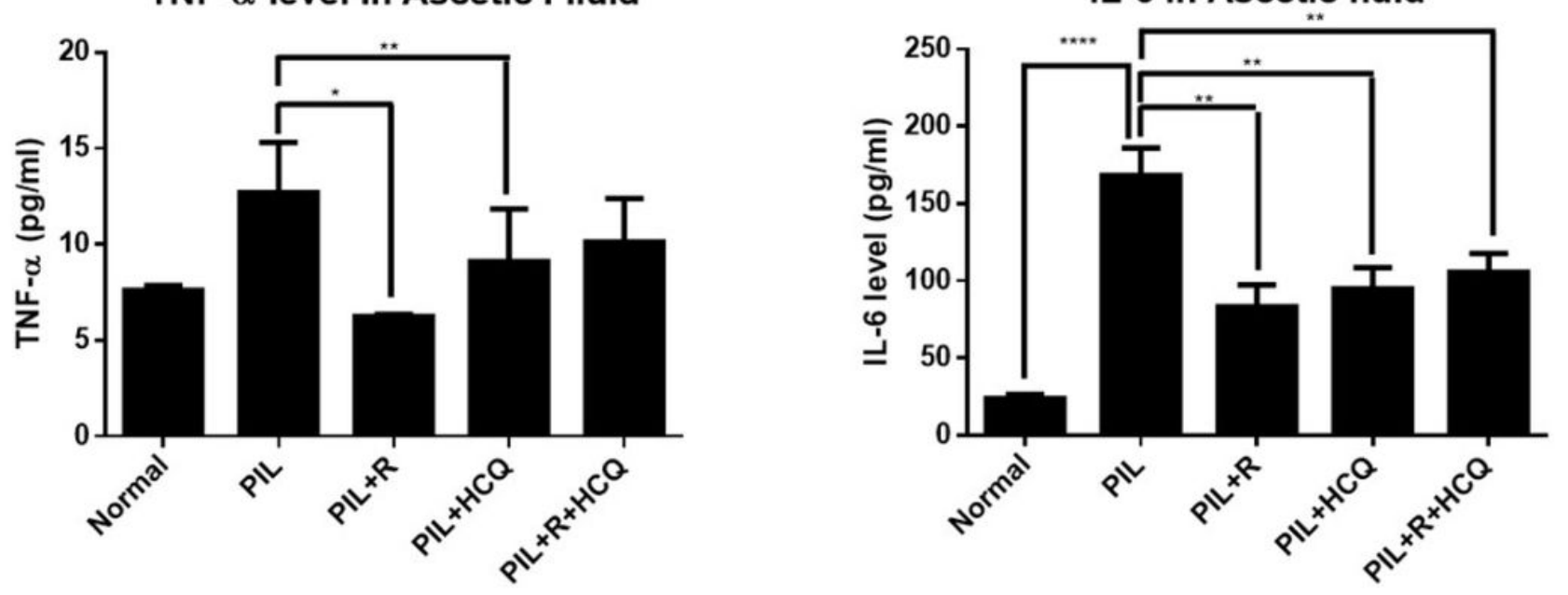

Figure 3

Analysis of autoantibodies and cytokines in mice with pristane-induced lupus.

Serum samples were collected for antinuclear antibody (ANA) and anti-dsDNA tests at 1, 3 and 6 months. Ascites fluid samples were collected for analysis of cytokines at 6 months. In the PIL mice, pristaneinduced increases in ANA titers were observed at 1 month and continued to increase thereafter until month 6 (Fig. 3A). Similarly, pristane-induced increases in anti-dsDNA antibodies were observed at 3 
months and continued to increase until month 6 (Fig. 3B). ANA and DNA titers were unchanged by treatments with HCQ and/or RESV. Pristane increased TNF- $a$ in ascites fluid at 6 months after PIL induction; HCQ or RESV treatment decreased TNF-a levels (Fig. 3C). Pristane also increased IL-6 levels in ascites fluid at 6 months after PIL induction, while HCQ and/or RESV treatment significantly decreased IL6 levels (Fig. 3D).

A

Taxonomy Summary: Family level B

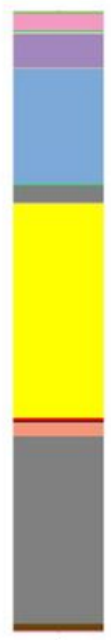

A

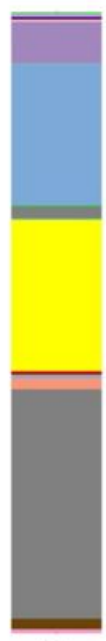

B

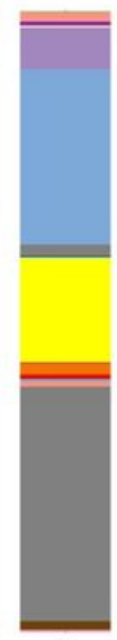

C

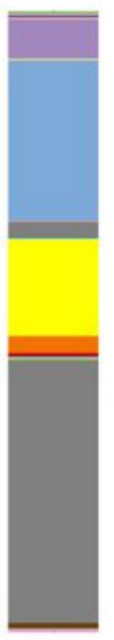

D

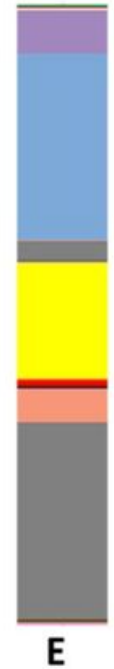

Firmicutes;Clostridia;Clostridiales;Lachnospiraceae Firmicutes;Bacilli;Lactobacillales;Lactobacillaceae

Bacteroidetes;Bacteroidia;Bacteroidales;Muribaculaceae

A: PIL_RESV+HCQ, B: PIL_RESV,

C: PIL_HCQ, D: PIL, E: Normal

Firmicutes

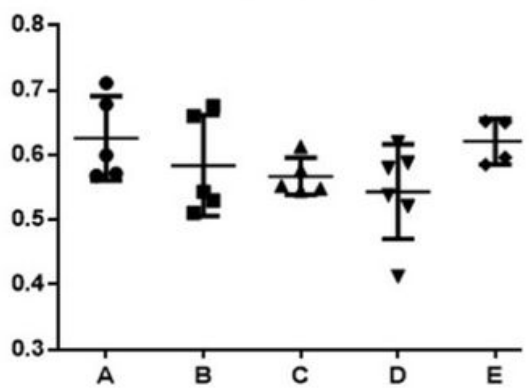

C

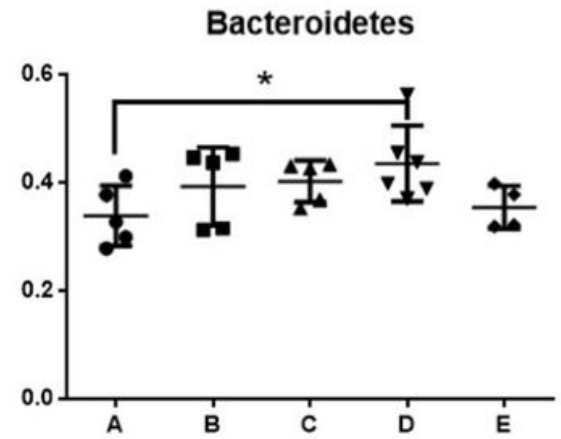

D
F/B ratio

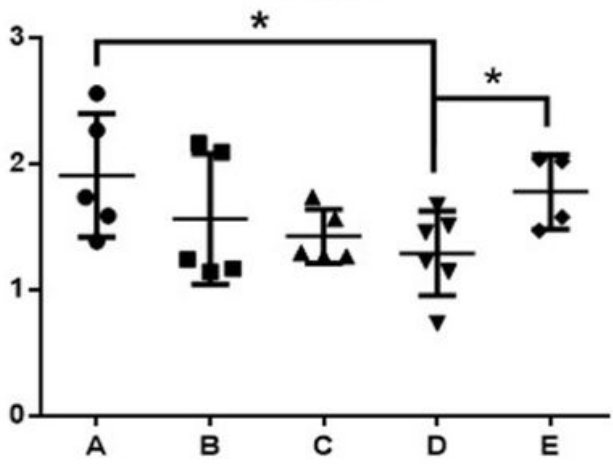

Figure 4

Analysis of Firmicutes and Bacteroidetes in mice with pristane-induced lupus.

A. Analyses of Firmicutes, Clostridia, Clostridiales, and Lachnospiraceae are indicated by blue coloring; Firmicutes, Bacilli, Lactobacillales, and Lactobacillaceae by yellow coloring; and Bacteroidetes, Bacteroidia, Bacteroidales, and Muribaculaceae by gray coloring. In the PIL mice, pristane decreased relative abundances of the Firmicutes and increased abundances of Bacteroidetes (Group D, Fig. 4A-C) compared with those in untreated control mice (Group E, Fig. 4A-C). HCQ showed a trend towards increasing Firmicutes phyla and decreasing Bacteroidetes phyla (Group C, Fig. 4 A-C) compared with pristane alone (Group D, Fig. 4A-C). Similar changes were observed in RESV-treated PIL mice (Group B, Fig. 4A-C) compared with PIL alone (Group D, Fig. 4A-C). Treatment with HCQ combined with RESV (Group A, Fig. 4A-C) significantly increased abundances of Firmicutes phyla and decreased abundances of Bacteroides phyla compared with PIL alone (Group D, Fig. 4A-C). A significant decrease in the Firmicutes/Bacteroidetes ( $F / B$ ) ratio was observed in PIL mice (Fig. 4D). Combined HCQ and RESV treatment in PIL mice significantly increased the $F / B$ ratio compared with PIL alone (Fig. 4D). 
Alpha Diversity

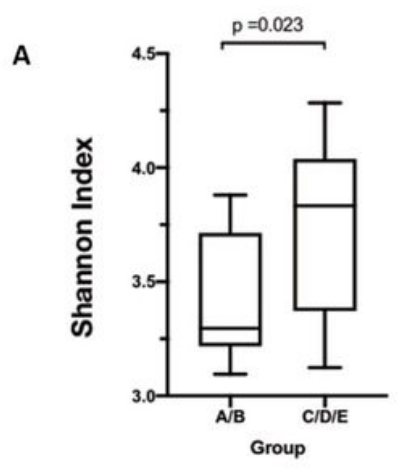

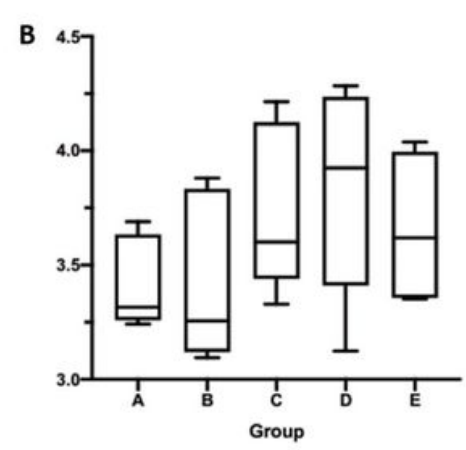

Beta Diversity

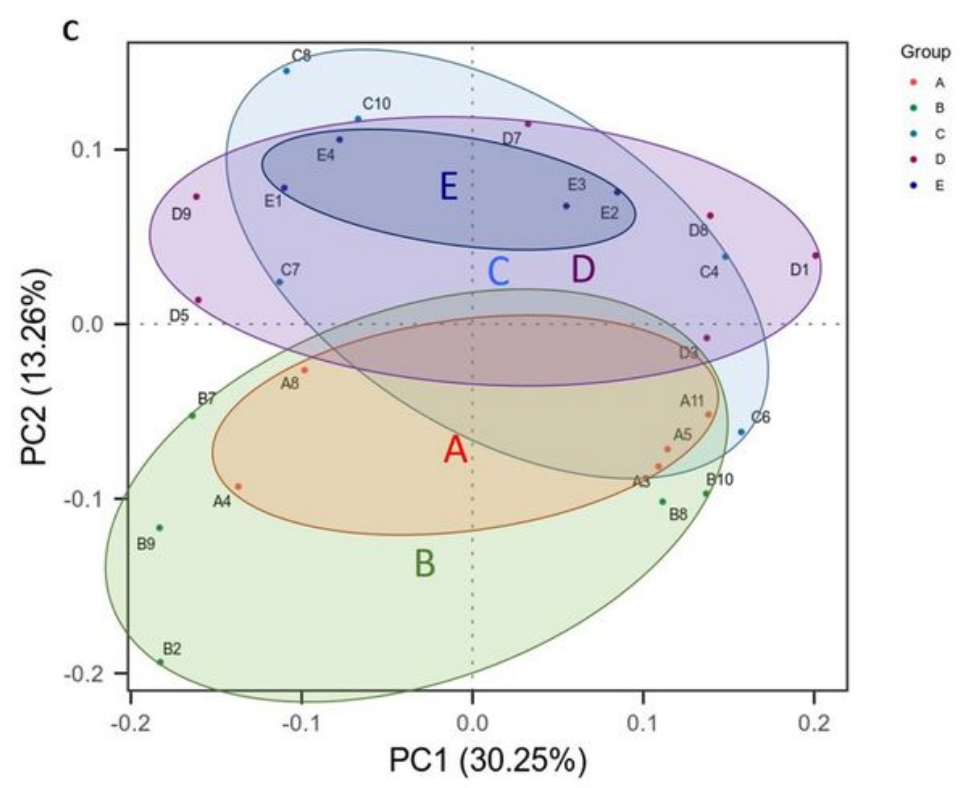

Figure 5

Measurements of alpha-diversity and beta-diversity.

Alpha-diversity measures in fecal samples based on the Shannon Index differed between groups that received RESV (Groups A and B, Fig. 5A) compared with groups that did not (Groups C, D and E, Fig. 5A; $p$ $=0.023)$. No statistically significant between-group differences were observed in Shannon Index scores (Groups A-E, Fig. 5B). Principal coordinates analysis (PCOA) of fecal samples revealed markedly different microbiome distributions in the groups that received RESV (Groups A and B, Fig. 5C) versus those that did not (Groups C, D and E, Fig. 5C). 